BMJ

Open

Gastroenterology

\title{
Scoping review of clinical practice guidelines on the management of benign liver tumours
}

\author{
Martijn P D Haring (D) ,' Frans J C Cuperus (D) , ${ }^{2}$ Evelien W Duiker (D), ${ }^{3}$ \\ Robbert J de Haas (D) , Vincent E de Meijer (i) ${ }^{1}$
}

To cite: Haring MPD,

Cuperus FJC, Duiker EW, et al. Scoping review of clinical practice guidelines on the management of benign liver tumours. BMJ Open Gastro 2021;8:e000592. doi:10.1136/ bmjgast-2020-000592

- Additional supplemental material is published online only. To view, please visit the journal online (http://dx.doi. org/10.1136/bmjgast-2020000592).

RJdH and VEdM contributed equally.

RJdH and VEdM are joint senior authors.

Received 19 December 2020 Accepted 18 July 2021
Check for updates

(C) Author(s) (or their employer(s)) 2021. Re-use permitted under CC BY-NC. No commercial re-use. See rights and permissions. Published by BMJ.

For numbered affiliations see end of article.

Correspondence to Dr Vincent E de Meijer; v.e.de.meijer@umcg.nl

\section{ABSTRACT}

Objective Benign liver tumours (BLT) are increasingly diagnosed as incidentalomas. Clinical implications and management vary across and within the different types of BLT. High-quality clinical practice guidelines are needed, because of the many nuances in tumour types, diagnostic modalities, and conservative and invasive management strategies. Yet, available observational evidence is subject to interpretation which may lead to practice variation. Therefore, we aimed to systematically search for available clinical practice guidelines on BLT, to critically appraise them, and to compare management recommendations. Design A scoping review was performed within MEDLINE, EMBASE, and Web of Science. All BLT guidelines published in peer-reviewed, and English language journals were eligible for inclusion. Clinical practice guidelines on BLT were analysed, compared, and critically appraised using the Appraisal of Guidelines, Research and Evaluation (AGREE II) checklist regarding hepatic haemangioma, focal nodular hyperplasia (FNH), and hepatocellular adenoma (HCA). Preferred Reporting Items for Systematic Reviews and Meta-Analyses recommendations (PRISMA) for scoping reviews were adhered to.

Results The literature search yielded unique 367 papers, 348 were excluded after screening of title/abstract, and 16 after full-text screening. Three guidelines were included: the American College of Gastroenterology (ACG; 2014), Brazilian Society of Hepatology (SBH; 2015), and European Association for the Study of the Liver (EASL; 2016). There was no uniformity in the assessment methods for grading and gravity of recommendations between guidelines. Among observed differences were: (1) indications for biopsy in all three tumours; (2) advices on contraceptive pills and follow-up in FNH and HCA; (3) use of an individualised approach to HCA; (4) absence of recommendations for treatment of HCA in men; and (5) approaches to HCA subtype identification on magnetic resonance imaging.

Conclusion Recognising differences in recommendations can assist in harmonisation of practice standards and identify unmet needs in research. This may ultimately contribute to improved global patient care.

\section{INTRODUCTION}

Hepatic incidentalomas are increasingly diagnosed due to the frequent use of

\section{Key points}

- Guidelines on benign liver tumours are necessary to prevent clinical practice variation and suboptima treatment

- Three guidelines on benign liver tumours provided several differences in recommendations regarding hepatocellular adenoma, focal nodular hyperplasia, and hepatic haemangioma

- Indications for biopsy in hepatocellular adenoma, focal nodular hyperplasia and hepatic haemangioma differed

- Life style advices on contraceptive pills and followup in focal nodular hyperplasia and hepatocellular adenoma varied

- Some guidelines did not provide specific recommendations for treatment of hepatocellular adenoma in men and differed in recommendations on subtype identification on MRI

- Recognising differences can assist in harmonisation of practice standards, identify unmet needs in research, and may ultimately contribute to improved global patient care

diagnostic imaging. These pathologies have a prevalence of about $15 \%$ in general but are observed in up to $30 \%$ of individuals older than 40 years. ${ }^{1-3}$ The majority of hepatic incidentalomas are benign and include hepatic cysts, focal fatty sparing and benign liver tumours (BLT). ${ }^{2}$ BLT comprise a heterogeneous group of tumours with distinct cellular origins, characterised by non-metastasizing, non-invasive behaviour. Most common BLT are hepatic haemangioma, focal nodular hyperplasia (FNH), and hepatocellular adenoma (HCA), which differ extensively in their clinical consequences. ${ }^{3}$

Hepatic haemangiomas are hypervascular tumours not at risk for malignant transformation. ${ }^{4}$ Rupture is extremely unlikely, often only after blunt trauma and is associated with high mortality. ${ }^{4}$ Large hepatic haemangiomas $(\geq 5 \mathrm{~cm})$ can cause abdominal pain and nausea by compression or, in rare cases, 
cause consumptive coagulopathy (Kasabach-Merritt syndrome). ${ }^{4}$

FNH are solitary, well-circumscribed, unencapsulated masses including a central fibrous scar and not at risk for haemorrhage or malignant transformation. ${ }^{5}$ Contraceptive pill (CP) use and pregnancy do not affect FNH size or number. ${ }^{6}$ Highest diagnostic sensitivity and specificity are obtained by hepatobiliary contrastenhanced magnetic resonance imaging (CE-MRI): respectively $92 \%-96.9 \%$ and $91 \%-100 \%$, irrespective of size. ${ }^{78}$ Contrast-enhanced ultrasound (CEUS) has been reported to improve the specificity of CE-MRI (especially when using extracellular contrast agents) in small $\mathrm{FNH}$ $(<3 \mathrm{~cm}) .{ }^{910}$

HCA are hypervascular tumours associated with potentially lethal haemorrhage $(10 \%)$ and may transform into hepatocellular carcinoma (5\%). ${ }^{11-13}$ Prolonged androgen exposure (CP, androgenic steroids, and obesity) is the major risk factor for HCA formation and growth. ${ }^{11} 1415 \mathrm{CP}$ cessation and weight loss can induce HCA regression. ${ }^{14} 16$ HCA subtypes are diagnosed through either immunohistochemistry or molecular analyses and have specific morphological and etiological features, clinical characteristics, and behaviours. ${ }^{12} 15$ Inflammatory HCA (I-HCA; 40\%-55\% of HCA) mainly co-occur with obesity and/or metabolic syndrome. ${ }^{15}$ Hepatocyte nuclear factor 1a (HNF1A) activated HCA (H-HCA; $30 \%-40 \%$ of HCA) rarely bleed or show malignant transformation. ${ }^{15}$ Adenomatosis $(\geq 10$ HCA) is associated with metabolic disorders such as HNF1A maturity onset diabetes of the young and glycogen storage disease (GSD). ${ }^{17} 18$ B-catenin activated HCA (b-HCA; 10\%) are at increased risk for malignant transformation to hepatocellular carcinoma (HCC) and more often occur in men. Importantly, half of b-HCA are hybrid b-catenin/inflammatory HCA. ${ }^{15} 19$ Invasive treatment is always warranted if HCA are diagnosed in men, as most HCA in men are b-HCA, and male sex is an independent additional driver of HCC formation. ${ }^{15}$ Two additional HCA subtypes with corresponding phenotype, sonic hedgehog, and roof plate spondin 2 HCA have been identified. ${ }^{1520}$ The remainder are characterised as unclassified HCA (U-HCA). Some reports have been able to differentiate HCA subtypes on CE-MRI, although no specific characteristics for b-HCA have yet been identified. ${ }^{21} 22$

The aforementioned intratumoral and intertumoral differences necessitate clear and consistent clinical practice guidelines to prevent (inter)national practice variation. Determining differences between current guidelines can provide a framework for practice standard harmonisation, identify unmet needs in research, and ultimately contribute to improved patient care. Until now, it is unclear how many clinical practice guidelines on BLT management are available, what the quality of available guidelines is, and to what extent management recommendations differ. Therefore, we aimed to systematically search for available clinical practice guidelines on BLT, to critically appraise them, and to compare management recommendations in a scoping review.

\section{METHODS}

A scoping literature review was performed including clinical practice guidelines on the management of BLT. Guidelines specifically developed for imaging or on gastroenterological pathologies in a specific context (ie, paediatric population or pregnancy) were excluded. The review was performed according to the extended Preferred Reporting Items for Systematic Reviews and Meta-Analyses recommendations for scoping reviews. ${ }^{23}$ No formal review protocol was drafted prior to execution of the study.

\section{Literature search}

A systematic literature search was performed by two investigators using appropriate prespecified search terms (online supplemental file 1) within the bibliographic databases of MEDLINE, EMBASE, and Web of Science, from inception, with the latest search on 31 March 2021. Only peer reviewed papers (no preprint) and only English articles were included. Reference lists of finally included papers were hand searched. Literature search and screening, and data extraction and appraisal were performed in duplicate by MPDH and VEdM. A third reviewer $(\mathrm{RJdH})$ was consulted for resolving any discrepancies.

\section{Data extraction and critical appraisal}

Guideline recommendations and recommendation strength were extracted and structured according to either recommendations in the applied systems of evidence grading or 'in text conclusions'. Oxford levels of evidence grade I was regarded as high-quality research, grade II-1 and II-2 as moderate quality, grade II-3 as low quality, and grade III as very low quality. ${ }^{24}$ Guidelines were appraised with the Appraisal of Guidelines, Research and Evaluation (AGREE II) checklist. ${ }^{25}$ AGREE II contains 23 items scored one (strongly disagree) to seven (strongly agree) points. Items span six domains: scope and purpose, stakeholder involvement, rigour of development, clarity of presentation, applicability, and editorial independence and an overall assessment. Scaled domain scores were calculated according to protocol by two reviewers. ${ }^{25}$ A third reviewer was consulted in for resolving any points of discussion between the two reviewers.

\section{RESULTS}

\section{Quantity and quality of evidence}

MEDLINE, EMBASE, and Web of Science queries provided 78, 189, and 176 results, respectively, leading to 367 original articles (figure 1). Seventy-six duplicates were removed. Screening of titles and abstracts resulted in exclusion of 348 publications. Full-text screening of the 19 remaining publications lead to further exclusion of 16 publications. Three guidelines were identified: American College of Gastroenterology (ACG) clinical guideline 'diagnosis and management of focal Liver lesions' (2014), 'diagnosis and treatment of benign liver nodules: Brazilian Society of Hepatology (SBH) 


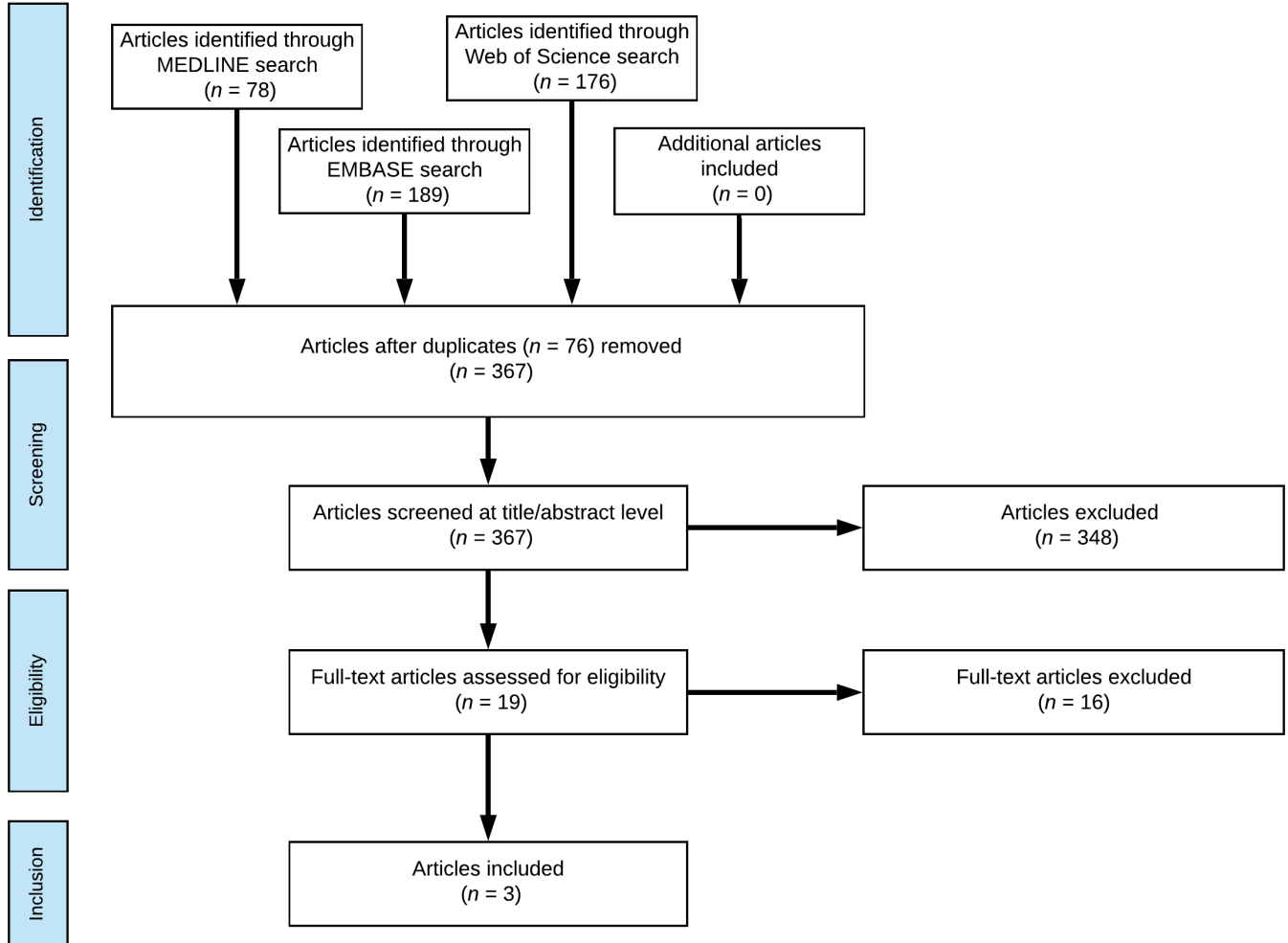

Figure 1 Flow chart of systematic literature search.

recommendations' (2015), and European Association for the Study of the Liver (EASL) 'clinical practice guideline on the management of benign liver tumors' (2016) ${ }^{26-28}$ Reference lists of the identified and included papers were hand searched, but no additional clinical practice guidelines could be identified.
The AGREE II domain scores favoured the EASL guideline in four out of six domains (figure 2, online supplemental file 2). ${ }^{25}$ Two guidelines indicated grade and gravity of recommendations (table 1). The ACG guideline used the four-level Grading of Recommendations Assessment, Development, and Evaluation system ${ }^{29}$ and

\section{Criticial appraisal of guidelines on benign liver tumors with AGREE II}

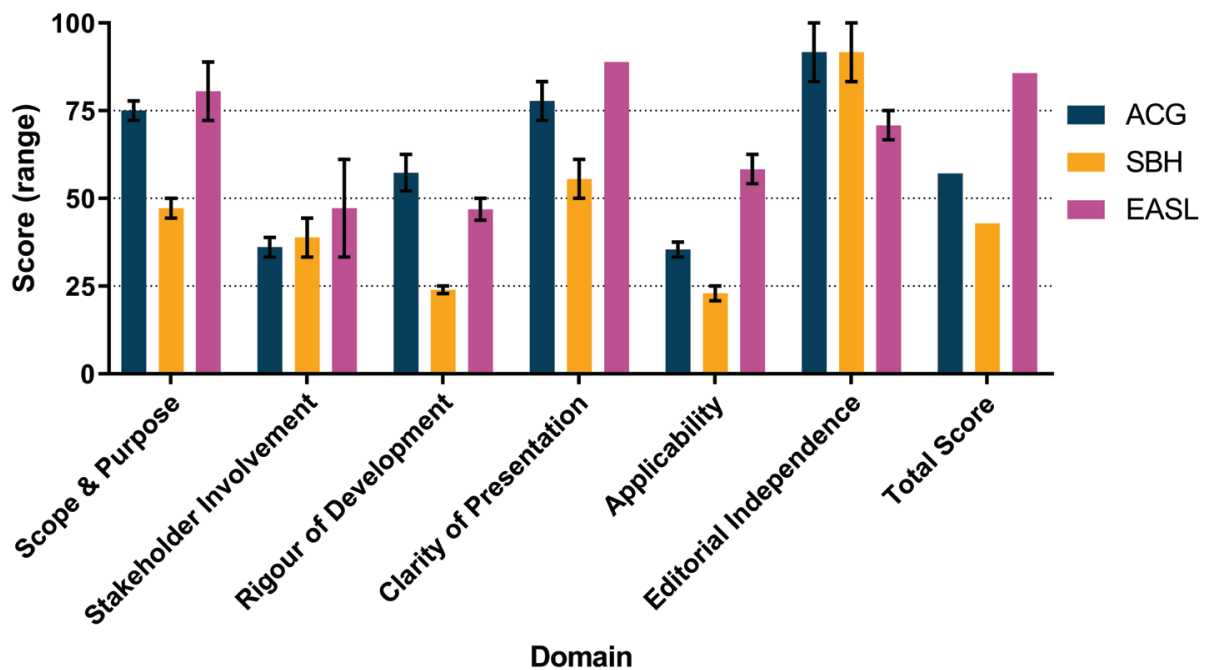

Figure 2 AGREE II scores of guidelines on benign liver tumours AGREE II scores per domain as assessed by two reviewers. Domain scores calculated as instructed in AGREE II protocol. ${ }^{25}$ ACG, American College of Gastroenterology; EASL, European Association for the Study of the Liver; SBH, Brazilian Society of Hepatology. 


\section{Table 1 Comparison of methodology in grading of evidence and strength of recommendation}

\begin{tabular}{|c|c|c|}
\hline \multicolumn{3}{|c|}{ System for grading of evidence } \\
\hline ACG $(2014)^{27}$ & SBH $(2015)^{26}$ & EASL $(2016)^{28}$ \\
\hline $\begin{array}{l}\text { High quality: further research unlikely to change confidence } \\
\text { of effect }\end{array}$ & $\begin{array}{l}\text { No grading system } \\
\text { applied }\end{array}$ & Grade I: randomised, controlled trials \\
\hline \multirow{2}{*}{$\begin{array}{l}\text { Moderate quality: further research likely to change the } \\
\text { confidence in the estimate of the effect and may change the } \\
\text { estimate }\end{array}$} & & $\begin{array}{l}\text { Grade II-1: controlled trials without } \\
\text { randomisation }\end{array}$ \\
\hline & & $\begin{array}{l}\text { Grade II-2: cohort or case-control } \\
\text { analytical series }\end{array}$ \\
\hline $\begin{array}{l}\text { Low quality: further research is very likely to change } \\
\text { confidence in the estimate of the effect and likely to change } \\
\text { the estimate }\end{array}$ & & $\begin{array}{l}\text { Grade II-3: multiple time series, } \\
\text { dramatic uncontrolled experiments }\end{array}$ \\
\hline Very low quality: any estimate of effect is very uncertain & & $\begin{array}{l}\text { Grade III: opinions of respected } \\
\text { authorities, descriptive epidemiology }\end{array}$ \\
\hline
\end{tabular}

\section{Type and strength of recommendation}

R Not applicable

S 'Factors influencing the strength of the recommendation include the quality of the evidence, presumed patientimportant outcomes, and cost'.

W 'Variability in preferences and values, or more uncertainty: more likely a weak recommendation is warranted.

Recommendation is made with less certainty: higher cost or resource consumption'.

t $\quad$ In text conclusion
Recommendations without Not applicable definition of gravity

Not applicable

Not applicable intervention clearly outweigh the undesirable effects or clearly do not'.

'The tradeoffs are less certain between the desirable and undesirable effects of an intervention'.

In text conclusion
In text conclusion

Blue = High level of evidence; Green = Moderate level of evidence; Orange = Low level of evidence; Red = Very low level of evidence . ACG, American College of Gastroenterology; EASL, European Association for the Study of the Liver; S, strong recommendation; SBH, Brazilian Society of Hepatology; W, weak recommendation/conditional recommendation.

used ACG guideline standards and Practice Parameters Committee guidance. The ACG guideline also stated used databases and search terms and specific author contributions. The EASL stated adoption from the GRADE system but practically used the five-level US Preventive Services Task Force scale with comparable definitions. ${ }^{30}$ The EASL and SBH guidelines did not specify search terms or used databases. None of the guidelines presented a flow chart of the literature review results. All guidelines provided both explicit recommendations and in text advices.

\section{Content of the guidelines}

The ACG guideline included literature up to June 2013 on both benign and malignant liver tumours. Discussed benign entities are: hepatic haemangioma, FNH, HCA, focal regenerative hyperplasia, simple hepatic cysts, biliary cystadenomas, polycystic liver disease, and hydatid cysts. $^{27}$ The SBH guideline did not mention a literature study timeframe; the most recent included publication dates September 2014. It includes hepatic haemangioma, FNH, HCA, simple hepatic cysts, hydatid cysts, cystadenomas, and polycystic liver disease. ${ }^{26}$ The EASL guideline did not mention the time frame of the literature study either but included studies published up to July $2015 .{ }^{28}$ It focused on hepatic haemangioma, FNH, and HCA. Nodular (or focal) regenerative hyperplasia is mentioned but referred to alternate reviews for recommendations on diagnostic features and management. It was the only guideline recommending use of a BLT multidisciplinary team consisting of a hepatologist, hepatobiliary surgeon, diagnostic and interventional radiologists, and pathologist.

\section{Hepatic haemangioma}

The ACG and SBH guidelines discouraged percutaneous needle biopsy due to bleeding risk, in contrast to the EASL guideline (table 2). Indications for surgical intervention differed slightly, as ACG guidelines included consideration of haemangiomas $\geq 10 \mathrm{~cm}$, while the other guidelines only included surgery for symptomatic tumours. Follow-up recommendations were similar in ACG and EASL guidelines. The SBH guideline recommended follow-up through ultrasound (US) in large haemangiomas and in pregnant patients. The SBH guideline was the only guideline to suggest liver transplantation as option for surgical treatment.

\section{Focal nodular hyperplasia}

Guidelines differed slightly in their formulation of recommendations for FNH (table 3). The ACG guideline recommended using MRI or CT for diagnostic confirmation, without specifying the modality or MR contrast agents. SBH and EASL guidelines concluded CE-MRI superiority for both FNH identification and FNH-HCA differentiation. The EASL guideline recommended biopsy for suspected $\mathrm{FNH} \geq 3 \mathrm{~cm}$ if diagnosis is doubtful after CE-MRI or if uncertain in $<3 \mathrm{~cm}$ tumours after CEUS. The ACG guideline recommended biopsy if FNH 


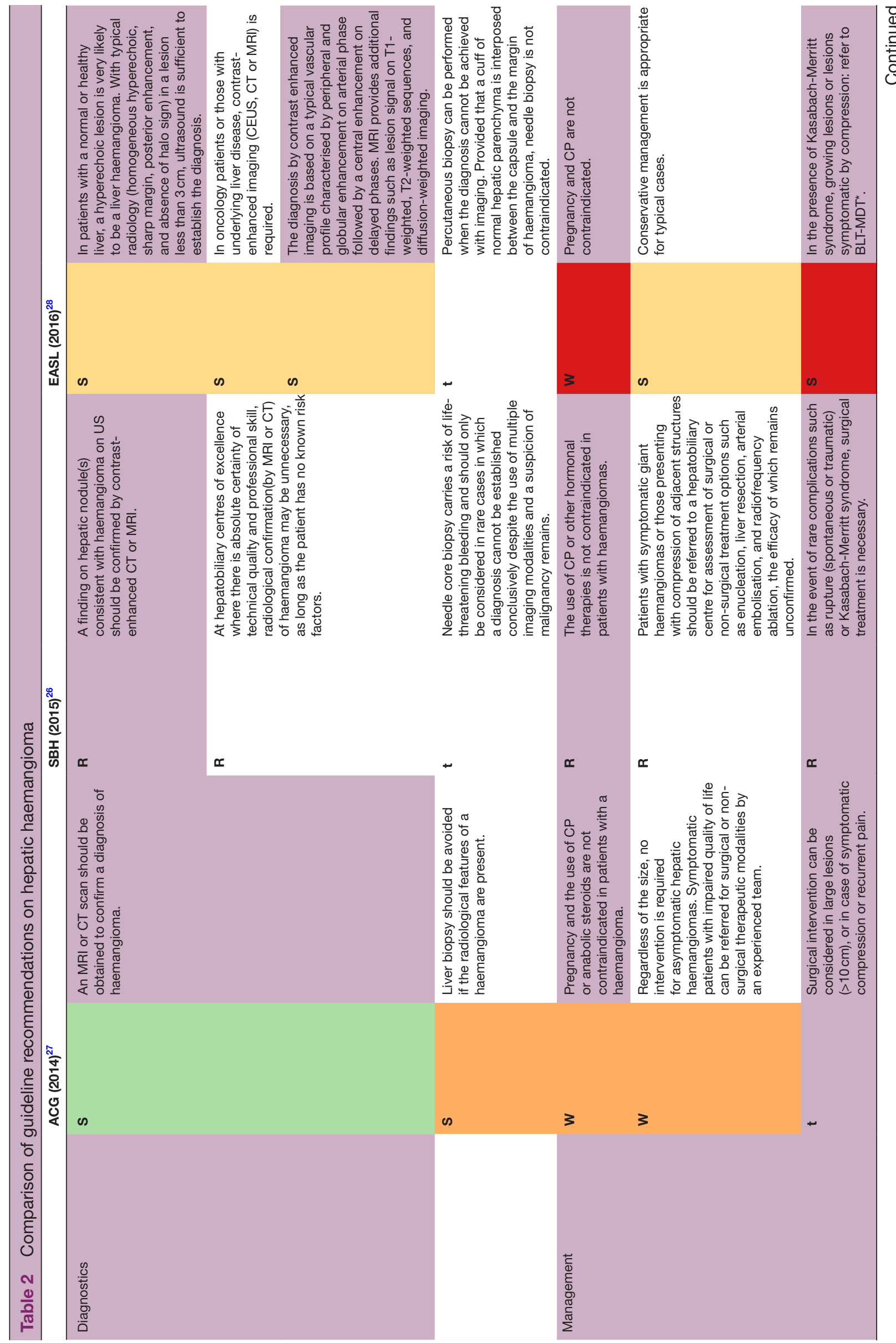




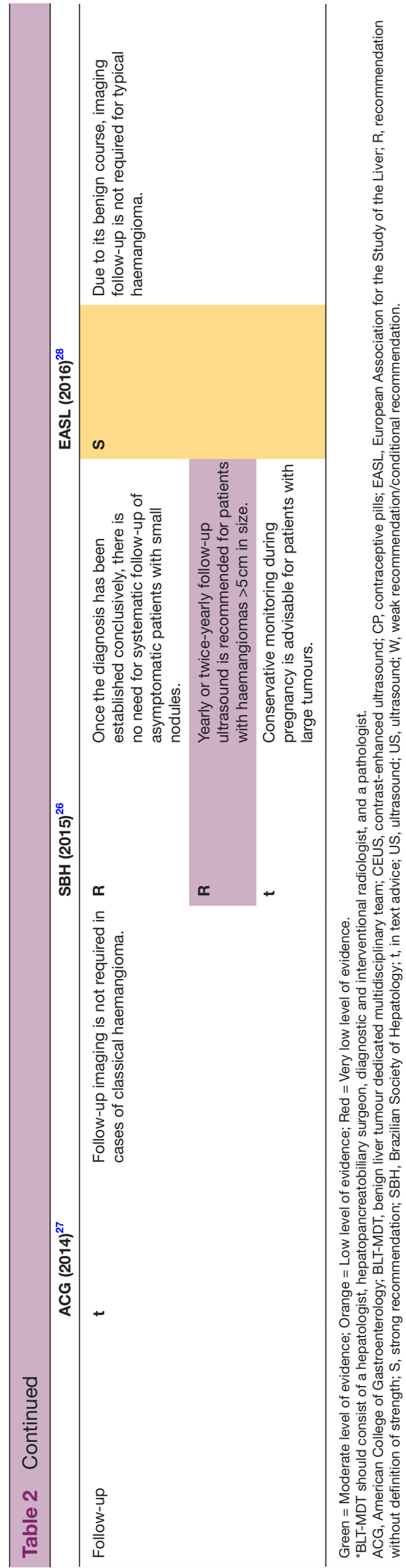

cannot be distinguished from HCA, without discussing the role of MRI and CEUS. The SBH did not discuss the role of biopsy in FNH.

Results regarding the use of CEUS (combined with MRI) for focal liver tumours were published prior to ACG and SBH guideline publication. ${ }^{31}$ Additional findings on CEUS use in FNH smaller than $3 \mathrm{~cm}$, though, were published after July 2013. ${ }^{9} 10$

The SBH guideline had no advices regarding CP use and concluded FNH's association with Oestrogens remains controversial. The SBH recommended follow-up every 6 months to 2 years, depending on tumour characteristics. The ACG recommended follow-up during 2-3 years in women when CP are continued. The EASL guideline did not recommend any follow-up unless concurring underlying vascular liver disease is present.

\section{Hepatocellular adenoma}

The guidelines differed moderately in HCA management recommendations (table 4). All guidelines recommended use of biopsies when imaging is inconclusive, and biopsy is necessitated for treatment decisions. Yet, none of the guidelines provided specific biopsy indications or a strict diagnostic workup. The EASL guideline preconditioned the consideration by a BLT multidisciplinary team prior to biopsy. All guidelines mentioned HCA subtype differentiation through MRI but differed in nuances. The ACG guideline described specific MRI characteristics for b-HCA and biopsy might prove unnecessary due to MRI HCA subtype characterisation. The SBH guideline noted identification of all HCA subtypes through MRI. The EASL guideline reported accurate characterisation of H-HCA or I-HCA, excluding b-HCA and U-HCA. B-HCA MRI features differed between ACG and EASL guidelines. The ACG guideline defined b-HCA as 'heterogeneous with no signal dropout on T1 out-ofphase sequences, isointense on $\mathrm{T} 1$ and $\mathrm{T} 2$ sequences, with strong arterial enhancement and delayed washout'. The EASL guideline observed b-HCA as 'mainly heterogeneously hyperintense on T2- and hypointense on T1-weighted sequences, with a central scar but no signal loss on chemical shift sequences'.

Conservative management was similar with regards to cessation of CP and anabolic steroids. The ACG guideline additionally included cessation of hormone-containing intra-uterine devices, in contrast to SBH and EASL guidelines. Only the EASL guideline recommended weight loss.

The ACG guideline did not mention male patients as specifically eligible for preemptive intervention. All guidelines described HCA diameter $\geq 5 \mathrm{~cm}$ as indication for (minimally) invasive intervention in females. Evaluation of prior lifestyle alteration effect was not included by the ACG and SBH guidelines. Management in the EASL guideline was individualised and gave recommendations based on patient sex, HCA size, HCA behaviour after lifestyle changes, and patients with multiple HCA. Results regarding the effect of weight loss on HCA diameter were 


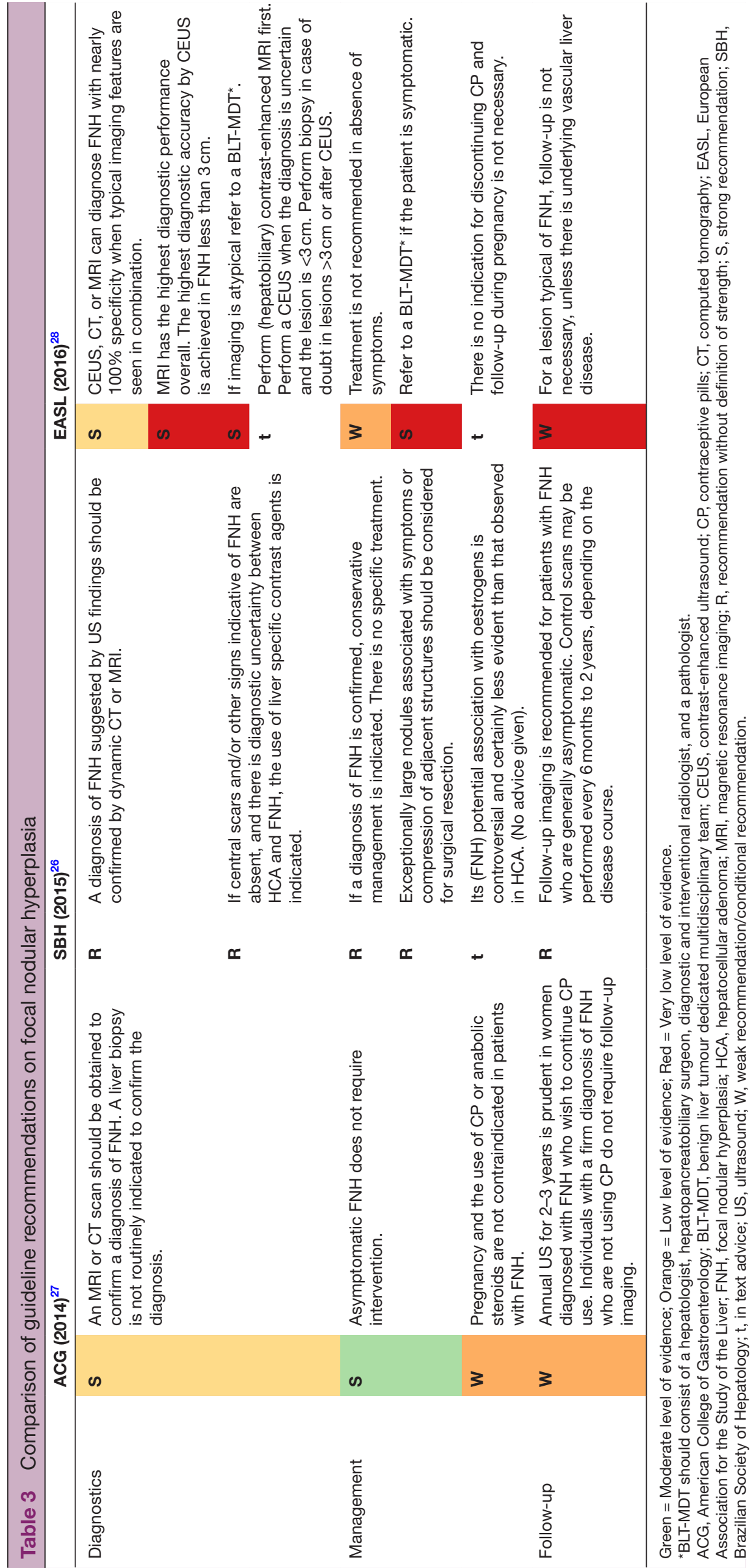




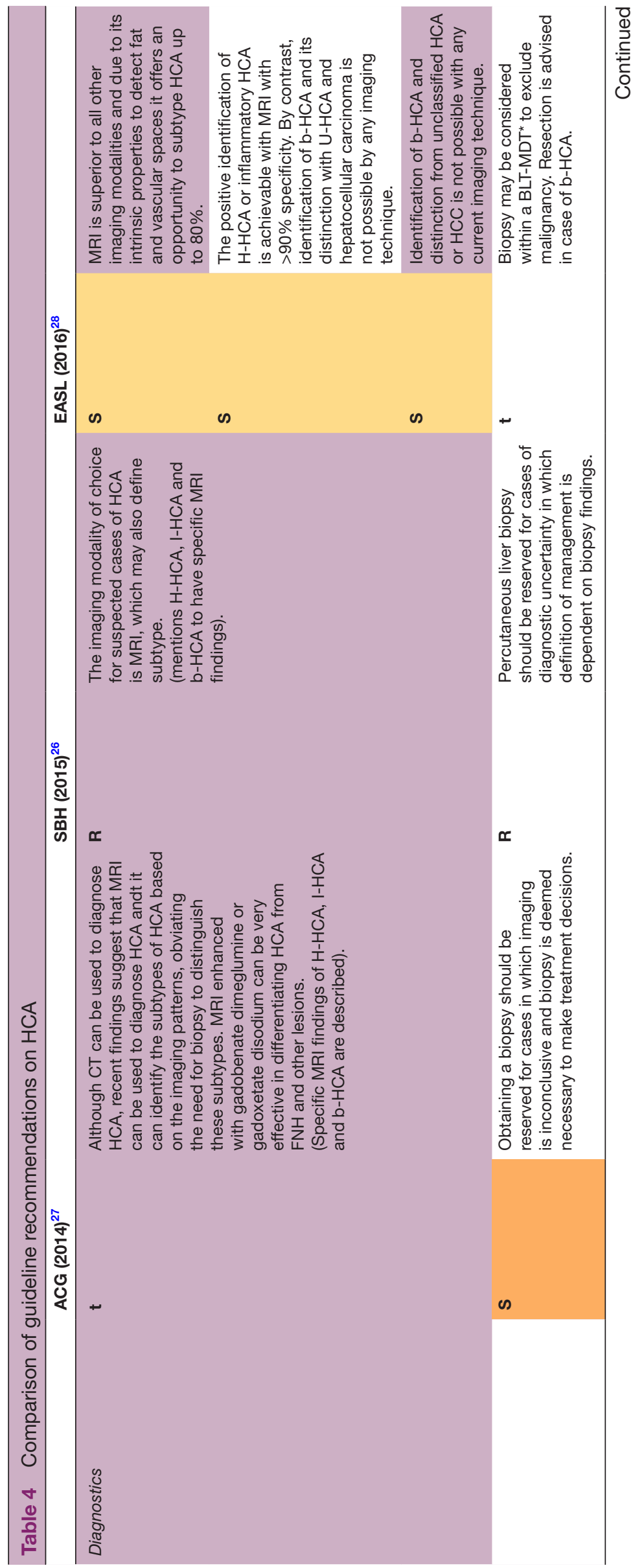




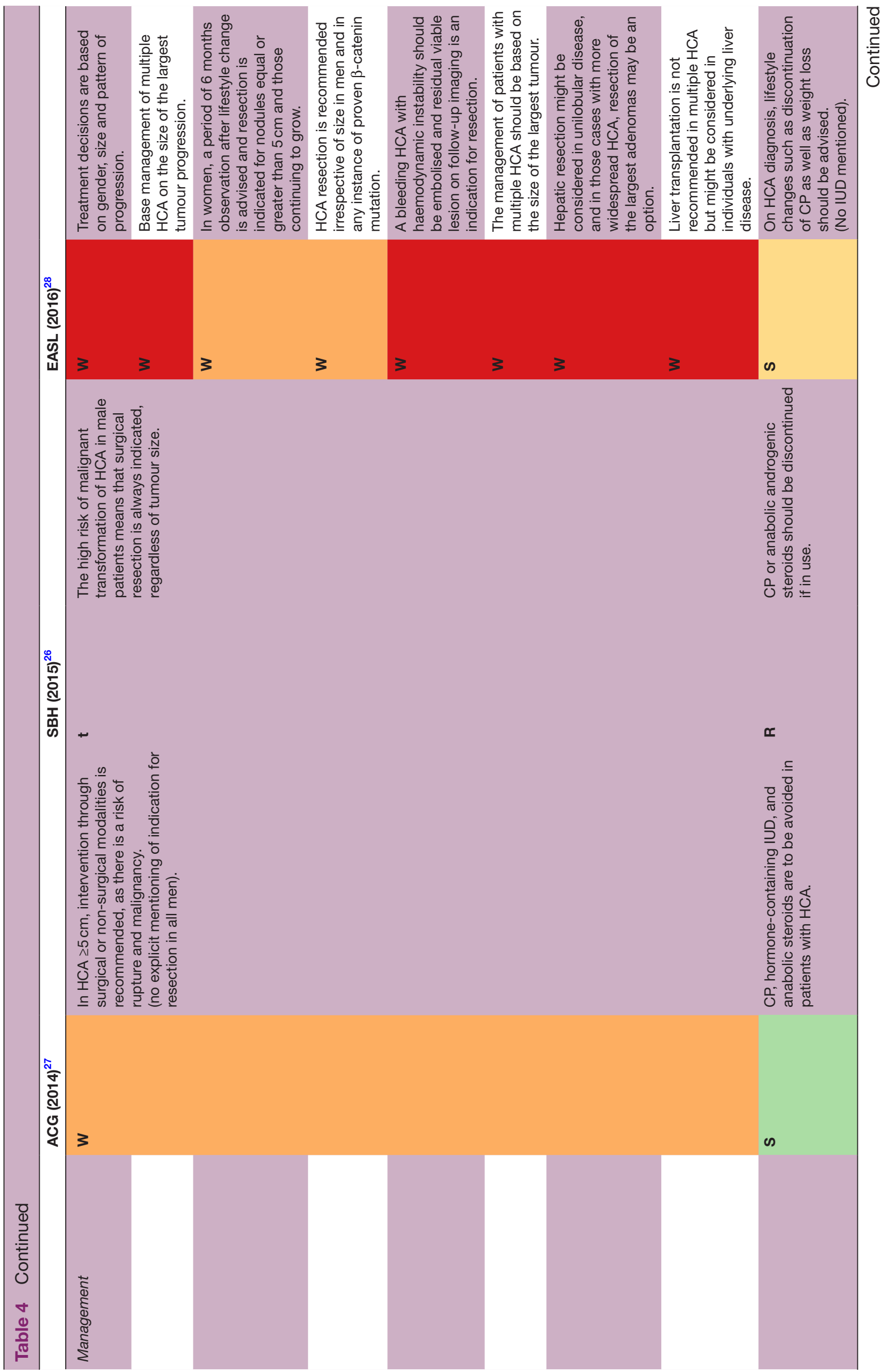




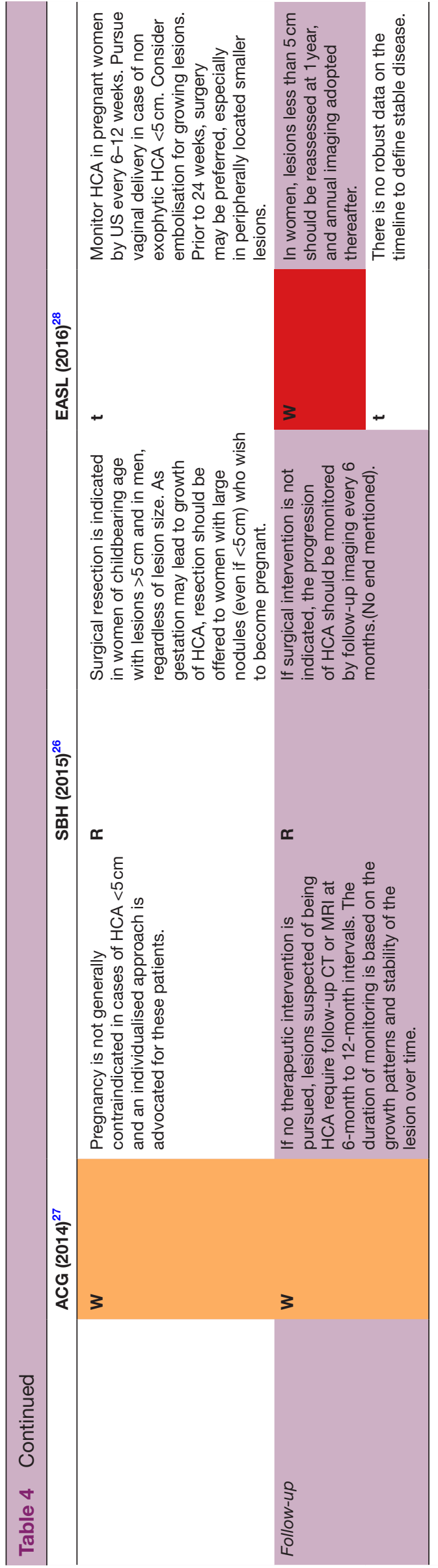

also reported after the publication of the ACG and SBH guidelines. ${ }^{14}$ The individualised strategy provided by the EASL guideline was proposed (but not yet clinical practice) in a review published within the ACG and SBH timelines. ${ }^{12}$ The SBH guideline advised resection of all HCA prior to pregnancy. The ACG and EASL recommended a case-by-case approach. The EASL guideline specified close follow-up by US, radiological or surgical intervention if growth occurs, and safety of vaginal delivery in nonexophytic HCA $<5 \mathrm{~cm}$. Follow-up intervals differed as the ACG advised 12 months, the SBH guideline 6 months and the EASL guideline 12 months after an initial 6-month evaluation of diameter after lifestyle changes. The ACG guideline advised liver transplantation only as definitive treatment of HCA in context of GSD, or as last resort in severe HCA-induced haemorrhage. The EASL states liver transplantation is not recommended in multiple HCA but might be considered in individuals with underlying liver disease.

\section{DISCUSSION}

The current review identified and indexed the currently available clinical practice guidelines on the management of BLT, critically appraised them and compared management recommendations. Three clinical practice guidelines were identified and included in the analysis, originating from the North American (ACG), South American $(\mathrm{SBH})$, and European (EASL) continent. Some differences in guideline quality were observed between guideline domains. Differences in the recommendations were identified in diagnostic workup, management, and follow-up of hepatic haemangioma, FNH, and HCA.

Multiple causes may explain the identified differences. First, ACG and SBH guidelines did solely focus on BLT but described (pre-)malignant tumours and cysts too. As the EASL guideline only focused on BLT, its authors had the possibility to provide a more in-depth overview. Second, discrepancies among the three guidelines could be a consequence of the moderate quality of the available and included (observational) evidence. This creates room for different-and equally justifiable-interpretations. Lastly, ACG and SBH guidelines were published up to 2 years before the EASL guideline. Novel insights emerged in this time-interval could explain differences in recommended treatment strategies.

There were different inclusions of available literature by the guidelines, leading to differences in recommendations on CEUS use. Interpretation of literature also differed between guidelines. For example, one report reported on 177 patients using oestrogen-containing $\mathrm{CP}$ for 9 years and excluded any influence of oestrogens on FNH behaviour. ${ }^{6}$ It was published within the scope of all guidelines, but only the EASL guideline completely dismissed FNH patients from interventions and follow-up. Safety of percutaneous biopsy in haemangioma also varied, with ACG and SBH guidelines discouraging it due to haemorrhage risk. No references regarding safety of biopsy are provided by the 
ACG guideline. The SBH guideline referred to two publications. $^{32} 33$ These, however, do not explicitly discourage biopsy. The study by Klotz et $a \ell^{32}$ discourages biopsy because hepatic angiosarcoma (1\% of all hepatic tumours) is part of the differential diagnosis, with significant bleeding risk. The other publication, by Caseiro-Alves et al, ${ }^{33}$ provides evidence supporting safety of haemangioma biopsy. The EASL guideline did not discourage biopsies. It refers to a paper dating from 1998 by Caldironi $e t a l,{ }^{34}$ which observed two minor bleedings in 114 biopsies.

Another example are differing recommendations on the follow-up of FNH. The SBH guideline recommends follow-up because of their cited risk of potential misdiagnosis of fibrolamellar HCC as FNH. ${ }^{35}{ }^{36}$ However, these publications used outdated imaging and histopathological techniques, and current diagnostics are highly capable in differentiating HCG from $\mathrm{FNH}^{8}{ }^{87-39}$ The ACG guideline advices a conservative stance due to rarity of FNH-induced HCC formation or hepatic rupture. ${ }^{40-42}$ However, it does not cite literature for its recommendation of follow-up of FNH in female patients using CP. The EASL guideline states there is insufficient evidence to support or refute elective surgery for $\mathrm{FNH}^{43}$ However, it emphasises the very low probability of FNH-induced complications. ${ }^{44} 45$

The extent to which the guidelines advise HCA subtype identification on MRI differed. The SBH guideline stated H-HCA, I-HCA, and b-HCA can be discriminated on MRI. This guideline referred to a retrospective study that described 34 I-HCA, 11 H-HCA, and 3 b-HCA. ${ }^{46}$ The cited study focused on gadolinium chelate (Dotarem), and although the authors described accurate characteristics for H-HCA and I-HCA, an insufficient number b-HCA cases were included to allow identification of discriminating features. The EASL guideline takes a more conservative stance and states that even though the subtype identification on imaging holds promise, future studies should prove feasibility for a wider application of MRI subtype differentiation of HCA than in the highly specialised centres. The EASL guideline mainly appreciates H-HCA and I-HCA as distinguishable on MRI and includes three other retrospective studies from 2008 to 2015 in addition to the paper included by the $\mathrm{SBH}^{46-49}$ The three additional papers also included $12 \mathrm{~b}-\mathrm{HCA}$ cases, of which six were investigated after gadoxetic acid (Primovist)-enhanced MRI. The ACG guideline states that biopsy for HCA subtype identification is obviated by MRIguided diagnostics, referring to a retrospective study from 2008 which included 15 H-HCA, 27 I-HCA, and 2 b-HCA cases. ${ }^{47}$ Multiple publications on this topic consulted by the EASL guidelines were not discussed in the ACG and SBH guidelines. ${ }^{46-48}$ A critical evaluation of the methodology of all the aforementioned imaging studies spans beyond the scope of the current review. Though, we opine that non-invasive HCA subtype identification can be considered when HCA demonstrate obvious signs of H-HCA or I-HCA. The current evidence does not allow for b-HCA discrimination, especially in light of the malignant potential which this subtype associates with.
Recommendations on HCA during pregnancy were limited in the included ACG guideline. Both EASL and the ACG guideline on liver disease during pregnancy recommend growing or $\mathrm{HCA} \geq 5 \mathrm{~cm}$ to be treated by radiological or surgical intervention. ${ }^{2850}$ The $\mathrm{SBH}$ guideline approached HCA more aggressively by advocating resection prior to pregnancy in all cases. All guidelines agreed on the safety of haemangiomas and $\mathrm{FNH}$ during pregnancy. Additional recommendations on liver disease during pregnancy, including BLT, can be found elsewhere..$^{50-52}$

Some liver transplantations have been performed for hepatocellular adenomatosis, yet this should not be applied standardly in the context of the current donor organ shortage and transplantation associated morbidity. ${ }^{53}$ Both ACG and EASL made limited recommendations on the role of liver transplantation. Both guidelines stated it should only be applied for GSD (associated adenomatosis) as an exception, though finally only warranted as it is a broader therapy for the carbohydrate metabolism dysfunction. ${ }^{54}$ The ACG guideline also suggested to use liver transplantation for severe HCAinduced haemorrhage, which has been successfully been performed. ${ }^{55}$ BLT are also known to occur in paediatric patients, but none of the guidelines provided recommendations on this subpopulation. ${ }^{56}$

The current manuscript included all available gastroenterology guidelines on BLT, but excluded specific radiology guidelines, or guidelines on subtopics that could potentially contain recommendations on BLT in a specific context (ie, pregnancy or in the paediatric setting). Another potential limitation is the subjective nature of the AGREE II questionnaire for critical appraisal. This may have introduced a potential risk of bias which, however, was at least in part mitigated by the use of two independent scoring researchers.

BLT are rare but increasingly observed as incidentalomas. Although being benign, some tumours may cause potential serious complications, necessitating clear and complete guidelines. The current inclusion of only three guidelines demonstrates the need of further development of guidance for clinicians. Ideally, novel guidelines would be drafted by multidisciplinary panels with representatives of all relevant specialty associations to ensure homogeneity on subtopics like imaging, need for pathology, and surgical interventions. Formulation of a global consensus statement is also needed. Differing designs of the healthcare systems could prevent a truly one-size-fits-all approach. Though, consensus could be attained through a Delphi method with participation of allied international associations to ensure elimination of potential treatment variation. Lastly, future drafting of guidelines could be performed according to AGREE or RIGHT reporting guidelines to ensure quality and comparability. ${ }^{5758}$

Guideline authors could provide a framework to ensure comparable strategies on major topics such as diagnostics or treatment, with the opportunity to adjust the guideline 
to local practice and preference. The ultimate aim of such an approach is patient care improvement, and optimal use of (limited) healthcare budgets. Second, analysis of BLT guidelines uncovered a potential future research agenda. Currently, none of the guidelines provided recommendations on HCA in patients with HNF1A-MODY or GSD, while although rare, these are known for their high HCA prevalence. ${ }^{17}$ Additionally, no large series on molecular HCA subtypes and behaviour in men have been performed, nor has the role of artificial intelligence in BLT characterisation extensively been explored.

Since publication of the EASL guideline, several HCA papers have been published which may carry significant consequences for future guidelines. One report observed the currently used 6-month period for evaluation of lifestyle alterations to be potentially too short for sufficient HCA regression, especially in large $\mathrm{HCA}{ }^{59}$ Another highly debated subject is the management of HCA prior to, during, and after pregnancy. A major prospective study observed sub- $5 \mathrm{~cm}$ HCA to be safe during pregnancy, while a combined cohort study and systematic review observed only HCA-induced haemorrhages in HCA $>6.5 \mathrm{~cm}$ and observed HCA to cause (lethal) postpartum haemorrhage in rare cases. ${ }^{52}{ }^{60} \mathrm{HCA}$ smaller than $5 \mathrm{~cm}$ have been observed safe to discharge from follow-up after the menopause. ${ }^{61}$ In addition, there has been reporting on the novel identification of sonic hedgehog activated HCA and roof plate-specific spondin 2 gene rearranged HCA, the increased application and accuracy of CEUS and MRI for diagnostics, occurrence of HCA in men prior to diabetic symptoms in HNF1A-MODY, and conservative and (minimally) invasive HCA management by transarterial embolisation. ${ }^{1720}{ }^{62-64}$ Next-generation sequencing of HCA in men revealed frequent change of HCA or HCC diagnosis and several b-HCA that were not diagnosed by immunohistochemistry, which could warrant a more prominent role for genetic sequencing in HCA (subtype) diagnostics. ${ }^{19}$ Regarding the minimally invasive treatment of symptomatic haemangioma, transarterial embolisation and lipiodolisation was observed to be safe and effective in a systematic review including 1284 pooled patients. ${ }^{65}$ These new insights might warrant an update of (harmonised) clinical practice guidelines in the near future.

Our observations might have influence on two important topics: (1) creation of global clinical practice uniformity and (2) identifying areas of future research. First, although comparing and analysing clinical practice guidelines may not directly benefit medical professionals or patients, our observations clearly show significant differences in BLT guideline design, content, and considerations between continents. This may encourage global professionals in expanding their scope when facing clinical dilemmas. Additionally, guideline authors and policy makers could take previously drafted guidelines into account when updating recommendations. This could create uniformity by raising global awareness of the differences in approaching various BLT.
In conclusion, three guidelines on BLT were identified, and several differences were identified on diagnostic workup and management of hepatic haemangioma, FNH, and HCA after comparison. These included: use of a dedicated BLT multidisciplinary team for management decisions, indications for biopsy, timing and duration of follow-up, conservative management of $\mathrm{FNH}$, diagnosis of HCA subtypes on MRI, and (conservative) management of HCA. These differences could lead to a practice variation, and thereby to varying outcomes. By recognising these differences, future research and debate should be focused on both harmonisation of clinical practice standards and remaining lacunas for BLT to achieve best patient care worldwide.

\section{Author affiliations}

${ }^{1}$ Department of Surgery, University Medical Centre Groningen, Groningen, The Netherlands

${ }^{2}$ Department of Hepatology and Gastroenterology, University Medical Centre Groningen, Groningen, The Netherlands

${ }^{3}$ Department of Medical Biology and Pathology, University Medical Centre Groningen, Groningen, The Netherlands

${ }^{4}$ Department of Radiology, University Medical Centre Groningen, Groningen, The Netherlands

Contributors No collaborators were involved in drafting the current manuscript, apart from the already registered coauthors.

Funding The authors have not declared a specific grant for this research from any funding agency in the public, commercial or not-for-profit sectors.

Competing interests None declared.

Patient consent for publication Not required.

Provenance and peer review Not commissioned; externally peer reviewed.

Data availability statement Data sharing not applicable as no datasets generated and/or analysed for this study. All data are publicly available through the search query included in the methods section of the article.

Supplemental material This content has been supplied by the author(s). It has not been vetted by BMJ Publishing Group Limited (BMJ) and may not have been peer-reviewed. Any opinions or recommendations discussed are solely those of the author(s) and are not endorsed by BMJ. BMJ disclaims all liability and responsibility arising from any reliance placed on the content. Where the content includes any translated material, BMJ does not warrant the accuracy and reliability of the translations (including but not limited to local regulations, clinical guidelines, terminology, drug names and drug dosages), and is not responsible for any error and/or omissions arising from translation and adaptation or otherwise.

Open access This is an open access article distributed in accordance with the Creative Commons Attribution Non Commercial (CC BY-NC 4.0) license, which permits others to distribute, remix, adapt, build upon this work non-commercially, and license their derivative works on different terms, provided the original work is properly cited, appropriate credit is given, any changes made indicated, and the use is non-commercial. See: http://creativecommons.org/licenses/by-nc/4.0/.

\section{ORCID iDs}

Martijn P D Haring http://orcid.org/0000-0003-4789-3910

Frans J C Cuperus http://orcid.org/0000-0001-6742-1510

Evelien W Duiker http://orcid.org/0000-0002-9450-5282

Robbert J de Haas http://orcid.org/0000-0002-4915-6781

Vincent E de Meijer http://orcid.org/0000-0002-7900-5917

\section{REFERENCES}

1 Gore RM, Pickhardt PJ, Mortele KJ, et al. Management of incidental liver lesions on CT: a white paper of the ACR incidental findings Committee. J Am Coll Radiol 2017;14:1429-37.

2 Koea JB. Hepatic incidentaloma: the rule of TENS. HPB 2013;15:379-83. 
3 Kaltenbach TE-M, Engler P, Kratzer W, et al. Prevalence of benign focal liver lesions: ultrasound investigation of 45,319 hospital patients. Abdom Radiol 2016;41:25-32.

4 Hoekstra LT, Bieze M, Erdogan D, et al. Management of giant liver hemangiomas: an update. Expert Rev Gastroenterol Hepatol 2013;7:263-8.

5 Roncalli M, Sciarra A, Tommaso LD, Di TL. Benign hepatocellular nodules of healthy liver: focal nodular hyperplasia and hepatocellular adenoma. Clin Mol Hepatol 2016;22:199-211.

6 Mathieu D, Kobeiter H, Maison P, et al. Oral contraceptive use and focal nodular hyperplasia of the liver. Gastroenterology 2000;118:560-4.

7 Grazioli L, Bondioni MP, Haradome H, et al. Hepatocellular adenoma and focal nodular hyperplasia: value of gadoxetic acid-enhanced MR imaging in differential diagnosis. Radiology 2012;262:520-9.

8 Suh $\mathrm{CH}$, Kim KW, Kim GY, et al. The diagnostic value of GdEOB-DTPA-MRI for the diagnosis of focal nodular hyperplasia: a systematic review and meta-analysis. Eur Radiol 2015;25:950-60.

9 Roche V, Pigneur F, Tselikas L, et al. Differentiation of focal nodular hyperplasia from hepatocellular adenomas with low-mechanicalindex contrast-enhanced sonography (CEUS): effect of size on diagnostic confidence. Eur Radiol 2015;25:186-95.

10 Bertin C, Egels S, Wagner M, et al. Contrast-enhanced ultrasound of focal nodular hyperplasia: a matter of size. Eur Radiol 2014;24:2561-71.

11 Edmondson HA, Henderson B, Benton B. Liver-Cell adenomas associated with use of oral contraceptives. N Engl J Med Overseas Ed 1976;294:470-2

12 Nault J-C, Bioulac-Sage P, Zucman-Rossi J. Hepatocellular benign tumors-from molecular classification to personalized clinical care. Gastroenterology 2013;144:888-902

13 Farges O, Ferreira N, Dokmak S, et al. Changing trends in malignant transformation of hepatocellular adenoma. Gut 2011;60:85-9.

14 Dokmak S, Belghiti J. Will weight loss become a future treatment of hepatocellular adenoma in obese patients? Liver Int 2015;35:2228-32.

15 Nault J-C, Couchy G, Balabaud C, et al. Molecular Classification of Hepatocellular Adenoma Associates With Risk Factors, Bleeding, and Malignant Transformation. Gastroenterology 2017;152:880-94.

16 Haring MPD, Gouw ASH, de Haas RJ, et al. The effect of oral contraceptive pill cessation on hepatocellular adenoma diameter: a retrospective cohort study. Liver Int 2019;39:905-13.

17 Haring MPD, Vriesendorp TM, Klein Wassink-Ruiter JS, et al. Diagnosis of hepatocellular adenoma in men before onset of diabetes in HNF1A-MODY: Watch out for winkers. Liver Int 2019;39:2042-5.

18 Calderaro J, Labrune P, Morcrette G, et al. Molecular characterization of hepatocellular adenomas developed in patients with glycogen storage disease type I. J Hepatol 2013;58:350-7.

19 van Rosmalen BV, Furumaya A, Klompenhouwer AJ, et al. Hepatocellular adenoma in men: a nationwide assessment of pathology and correlation with clinical course. Liver Int 2021:liv.14989.

20 Longerich T, Endris V, Neumann O, et al. RSPO2 gene rearrangement: a powerful driver of $\beta$-catenin activation in liver tumours. Gut 2019;68:1287-96.

21 Bise S, Frulio N, Hocquelet A, et al. New MRI features improve subtype classification of hepatocellular adenoma. Eur Radiol 2019;29:2436-47

22 Auer TA, Fehrenbach U, Grieser C, et al. Hepatocellular adenomas: is there additional value in using Gd-EOB-enhanced MRI for subtype differentiation? Eur Radiol 2020;30:1-10.

23 Tricco AC, Lillie E, Zarin W, et al. PRISMA extension for scoping reviews (PRISMA-ScR): checklist and explanation. Ann Intern Med 2018;169:467-73.

24 OCEBM Levels of Evidence Working Group. The Oxford levels of evidence 2. Oxford centre for evidence-based medicine, 2011. Available: https://www.cebm.net/index.aspx?o $=5653$

25 Brouwers MC, Kho ME, Browman GP, et al. Agree II: advancing Guideline development, reporting and evaluation in health care. $J$ Clin Epidemiol 2010;63:1308-11.

26 Strauss E, Ferreira AdeSP, França AVC, et al. Diagnosis and treatment of benign liver nodules: Brazilian Society of hepatology (SBH) recommendations. Arq Gastroenterol 2015;52:47-54.

27 Marrero JA, Ahn J, Rajender Reddy K, Reddy RK, et al. Acg clinical guideline: the diagnosis and management of focal liver lesions. Am Gastroenterol 2014;109:1328-47.

28 European Association for the Study of the Liver (EASL). EASL clinical practice guidelines on the management of benign liver tumours. $J$ Hepatol 2016;65:386-98.
29 Guyatt GH, Oxman AD, Vist GE, et al. Grade: an emerging consensus on rating quality of evidence and strength of recommendations. BMJ 2008;336:924-6.

30 Lawrence RS. Guide to clinical preventive services: report of the U.S. preventive services Task force. 263. DIANE Publishing, 1989.

31 Soussan M, Aubé C, Bahrami S, et al. Incidental focal solid liver lesions: diagnostic performance of contrast-enhanced ultrasound and MR imaging. Eur Radiol 2010;20:1715-25.

32 Klotz T, Montoriol P-F, Da Ines D, et al. Hepatic haemangioma: common and uncommon imaging features. Diagn Interv Imaging 2013;94:849-59.

33 Caseiro-Alves F, Brito J, Araujo AE, et al. Liver haemangioma: common and uncommon findings and how to improve the differential diagnosis. Eur Radiol 2007;17:1544-54

34 Caldironi MW, Mazzucco M, Aldinio MT. [Echo-guided fine-needle biopsy for the diagnosis of hepatic angioma. A report on 114 cases] Minerva Chir 1998:53:505-9.

35 Imkie M, Myers SA, Li Y, et al. Fibrolamellar hepatocellular carcinoma arising in a background of focal nodular hyperplasia: a report of 2 cases. J Reprod Med 2005;50:633-7.

36 Hamrick-Turner JE, Shipkey FH, Cranston PE. Fibrolamellar hepatocellular carcinoma: Mr appearance mimicking focal nodular hyperplasia. J Comput Assist Tomogr 1994;18:301-4.

37 Rebouissou S, Bioulac-Sage P, Zucman-Rossi J. Molecular pathogenesis of focal nodular hyperplasia and hepatocellular adenoma. J Hepatol 2008;48:163-70.

38 Bioulac-Sage P, Laumonier H, Rullier A, et al. Over-expression of glutamine synthetase in focal nodular hyperplasia: a novel easy diagnostic tool in surgical pathology. Liver Int 2009;29:459-65.

39 Rebouissou S, Couchy G, Libbrecht L, et al. The beta-catenin pathway is activated in focal nodular hyperplasia but not in cirrhotic FNH-like nodules. J Hepatol 2008;49:61-71.

40 Haubert L, Yearsley M, Bloomston M. Hepatocellular carcinoma arising within focal nodular hyperplasia. Am Surg 2010;76:335-6.

41 Rahili A, Cai J, Trastour C, et al. Spontaneous rupture and hemorrhage of hepatic focal nodular hyperplasia in lobus caudatus. $J$ Hepatobiliary Pancreat Surg 2005;12:138-42.

42 Demarco MP, Shen P, Bradley RF, et al. Intraperitoneal hemorrhage in a patient with hepatic focal nodular hyperplasia. Am Surg 2006;72:555-9.

43 Colli A, Fraquelli M, Massironi S, et al. Elective surgery for benign liver tumours. Cochrane Database Syst Rev 2007:CD005164.

44 D'Halluin V, Vilgrain V, Pelletier G. Evolution naturelle de I'hyperplasie nodulaire focale. Etude rétrospective de 44 cas. Gastroenterologie Clinique et Biologique 2001;25:1008-10.

45 Perrakis A, Demir R, Müller V, et al. Management of the focal nodular hyperplasia of the liver: evaluation of the surgical treatment comparing with observation only. Am J Surg 2012;204:689-96.

46 Ronot M, Bahrami S, Calderaro J, et al. Hepatocellular adenomas: accuracy of magnetic resonance imaging and liver biopsy in subtype classification. Hepatology 2011;53:1182-91.

47 Laumonier H, Bioulac-Sage P, Laurent C, et al. Hepatocellular adenomas: magnetic resonance imaging features as a function of molecular pathological classification. Hepatology 2008;48:808-18.

48 van Aalten SM, Thomeer MGJ, Terkivatan T, et al. Hepatocellular adenomas: correlation of Mr imaging findings with pathologic subtype classification. Radiology 2011;261:172-81.

49 Ba-Ssalamah A, Antunes C, Feier D, et al. Morphologic and molecular features of hepatocellular adenoma with gadoxetic acidenhanced MR imaging. Radiology 2015;277:104-13.

50 Tran TT, Ahn J, Reau NS. Acg clinical guideline: liver disease and pregnancy. Am J Gastroenterol 2016;111:176-94.

51 Sarkar M, Brady CW, Fleckenstein J, et al. Reproductive health and liver disease: practice guidance by the American association for the study of liver diseases. Hepatology 2021;73:318-65

52 Haring MPD, Spijkerboer CS, Cuperus FJC, et al. Behavior and complications of hepatocellular adenoma during pregnancy and puerperium: a retrospective study and systematic review. HPB 2021 doi:10.1016/j.hpb.2021.04.019. [Epub ahead of print: 28 Apr 2021].

53 Chiche L, David A, Adam R, et al. Liver transplantation for adenomatosis: European experience. Liver Transpl 2016;22:516-26.

54 Reddy SK, Austin SL, Spencer-Manzon M, et al. Liver transplantation for glycogen storage disease type la. J Hepatol 2009;51:483-90.

55 Santambrogio R, Marconi AM, Ceretti AP, et al. Liver transplantation for spontaneous intrapartum rupture of a hepatic adenoma. Obstet Gynecol 2009;113:508-10.

56 Fuchs J, Warmann SW, Urla C, et al. Management of benign liver tumors. Semin Pediatr Surg 2020;29:150941.

57 Brouwers MC, Kerkvliet K, Spithoff K, et al. The agree reporting checklist: a tool to improve reporting of clinical practice guidelines. BMJ 2016;352:i1152. 
58 Chen Y, Yang K, Marušic A, et al. A reporting tool for practice guidelines in health care: the right statement. Ann Intern Med 2017;166:128-32.

59 Klompenhouwer AJ, Bröker MEE, Thomeer MGJ, et al. Retrospective study on timing of resection of hepatocellular adenoma. $\mathrm{Br} J$ Surg 2017;104:1695-703.

60 Gaspersz MP, Klompenhouwer AJ, Broker MEE, et al. Growth of hepatocellular adenoma during pregnancy: a prospective study. $J$ Hepatol 2020;72:119-24.

61 Klompenhouwer AJ, Sprengers D. A cohort study of hepatocellular adenoma in post-menopausal women: can follow-up be safely terminated? J Hepatol 2016;65:1163-70.
62 Nault J-C, Couchy G, Caruso S, et al. Argininosuccinate synthase 1 and periportal gene expression in sonic hedgehog hepatocellular adenomas. Hepatology 2018;68:964-76.

63 van Rosmalen BV, Klompenhouwer AJ, de Graeff JJ, et al. Safety and efficacy of transarterial embolization of hepatocellular adenomas. Br J Surg 2019;106:1362-71.

64 Bröker MEE, Taimr P, de Vries M, et al. Performance of contrastenhanced sonography versus MRI with a liver-specific contrast agent for diagnosis of hepatocellular adenoma and focal nodular hyperplasia. AJR Am J Roentgenol 2020;214:1-9.

65 Furumaya A, van Rosmalen BV, Takkenberg RB, et al. Transarterial (Chemo-)Embolization and lipiodolization for hepatic haemangioma. Cardiovasc Intervent Radiol 2019;42:800-11. 